\title{
Design of hyperboloid structures
}

\begin{abstract}
In this paper, we present the design of hyperboloid structures and techniques for hyperboloid fitting which are based on minimizing the sum of the squares of the geometric distances between the noisy data and the hyperboloid. The literature often uses "orthogonal fitting" in place of "best fitting". For many different purposes, the best-fit hyperboloid fitting to a set of points is required. Algebraic fitting methods solve the linear least squares (LS) problem, and are relatively straightforward and fast. Fitting orthogonal hyperboloid is a difficult issue. Usually, it is impossible to reach a solution with classic LS algorithms. Because they are often faced with the problem of convergence. Therefore, it is necessary to use special algorithms e.g. nonlinear least square algorithms. We propose to use geometric fitting as opposed to algebraic fitting. Hyperboloid has a complex geometry as well as hyperboloid structures have always been interested. The two main reasons, apart from aesthetic considerations, are strength and efficiency.
\end{abstract}

Volume 3 Issue $6-2017$

\author{
Sebahattin Bektas \\ Faculty of Engineering, Ondokuz Mayis University, Turkey
}

Correspondence: Sebahattin Bektas, Ondokuz Mayis

University, Faculty of Engineering, Geomatics Engineering, 55139

Samsun,Turkey, Email sbektas@omu.edu.tr

Received: October II, 2017| Published: December 27, 2017

Keywords: hyperboloid structure, design of hyperboloid, orthogonal fitting, algebraic fitting, nonlinear least square problem

\section{Introduction}

The industrial manufacturing is making more widespread use of three-dimensional object recognition techniques. The shapes of many $3 \mathrm{D}$ objects can be described using some basic surface elements. Many of these basic surface elements are quadric surfaces, such as spheres and cylinders. For some industrial parts, the surface located at the junction of two other surface elements can often be regarded as either a hyperbolic surface (concave) or a parabolic surface (convex). Therefore, the recognition of hyperboloids and paraboloids is necessary for the recognition or inspection of such kinds of 3D objects. The objective of surface fitting is to fit some kind of mathematical model, such as an equation under a certain kind of coordinate system, to the sample data. For centered quadric surfaces, such an equation can be expressed in a form where fit parameters have a clear geometric meaning, such as a distance or a rotation angle Min et al., ${ }^{1}$ As hyperboloid structures are double curved, that is simultaneously curved in opposite directions, they are very resistant to buckling. This means that you can get away with far less material than you would otherwise need, making them very economical. Single curved surfaces, for example cylinders, have strengths but also weaknesses. Double curved surfaces, like the hyperboloids in question, are curved in two directions and thus avoid these weak directions. This means that you can get away with far less material to carry a load, which makes them very economical. The second reason, and this is the magical part, is that despite the surface being curved in two directions, it is made entirely of straight lines. Apart from the cost savings of avoiding curved beams or shuttering, they are far more resistant to buckling because the individual elements are straight URL-1, URL-2. This is an interesting paradox: you get the best local buckling resistance because the beams are straight and the best overall buckling resistance because the surface is double curved. Hyperboloid structures cunningly combine the contradictory requirements into one form. The hyperboloid is the design standard for all nuclear cooling towers and some coal-fired power plants. It is structurally sound and can be built with straight steel beams.
When designing these cooling towers, engineers are faced with two problems:

i. The structure must be able to withstand high winds and

ii. They should be built with as little material as possible (Figure 1).

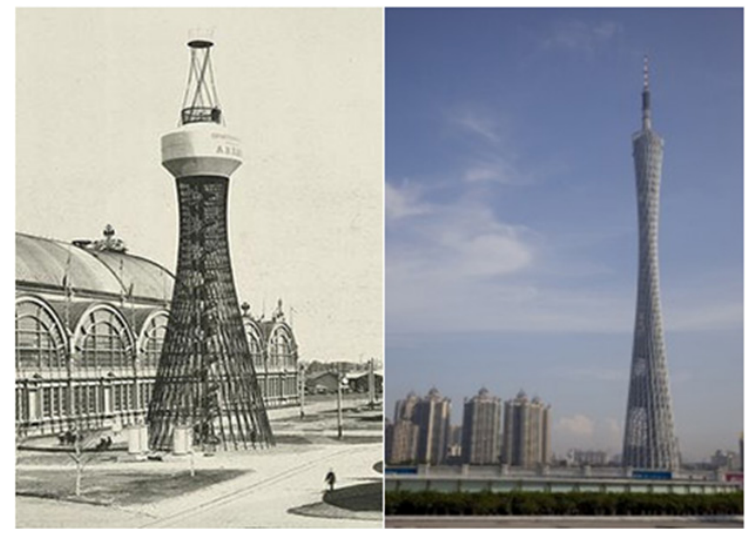

Figure I Towers: a) Shukhov Tower Nizhny Novgorod I896; b) Canton Tower, Guangzhou, China Kenny Ip.

The hyperbolic form solves both of these problems. For a given diameter and height of a tower and a given strength, this shape requires less material than any other form. The pioneer of hyperboloidal structures is the remarkable Russian engineer V Shukhov (18531939) who, among other accomplishments, built a hyperboloidal water tower for the 1896 industrial exhibition in Nizhny Novgorod. Hyperboloidal towers can be built from reinforced concrete or as a steel lattice, and is the most economical such structure for a given diameter and height. The roof of the McDonnell Plantarium in St. Louis, the Brasilia Cathedral and the Kobe Port tower are a few recent examples of hyperboloidal structures. The most familiar use, however, is in cooling towers used to cool the water used for the condensers of a steam power plant, whether fuel burning or nuclear. The bottom of the 
tower is open, while the hot water to be cooled is sprayed on wooden baffles inside the tower. Potentially, the water can be cooled to the wet bulb temperature of the admitted air. Natural convection is established due to the heat added to the tower by the hot water. If the air is already of moderate humidity when admitted, a vapor plume is usually emitted from the top of the tower. The ignorant often call this plume "smoke" but it is nothing but water. Smokestacks are the high, thin columns emitting at most a slight haze. The hyperboloidal cooling towers have nothing to do with combustion or nuclear materials. Two such towers can be seen at the Springfield Nuclear Plant on The Simpsons. The large coal facility at Didcot, UK also has hyperboloidal cooling towers easily visible to the north of the railway west of the station. Hyperboloidal towers of lattice construction have the great advantage that the steel columns are straight URL-1. ${ }^{1}$ For hyperboloid application please refers to URL-1, URL-2.,3 The paper has six parts. We will first give some general information about hyperboloid structures, the basic definition of Hyperboloid starts with giving mathematical equations to explain the concepts. Then it reviews the extend literature relevant to hyperboloid fitting. To show how to best fitting Hyperboloid, are carried out, we solve this problem separately: algebraic direct fitting Hyperboloid and the best fitting Hyperboloid. The efficacy of the new algorithms is demonstrated through simulations. The paper concludes with a discussion of theoretical and managerial implications and directions for further research. Unfortunately, best fitting hyperboloid has not been discussed in literature. However, most of the few fitting techniques in the literature are algebraic fitting are not orthogonal fitting Andrews et al. ${ }^{4}$ There are some published methods that focus on this topic. Hall ${ }^{5}$ approach is a least squares based approach to recover the parameters of quadric surfaces from depth maps. Although parameter recovery for quadric surfaces might seem to require a nonlinear method, the quadric coefficients can be recovered by least squares and then the geometric parameters of the surface can be recovered using Hall ${ }^{5}$ approach. $\mathrm{Cao}^{6}$ method is another pure numerical approach which emphasizes minimization of overall errors represented by a certain kind of approximate orthogonal distance. Their approach uses the iterative Newton method to perform nonlinear optimization. Their method may terminate at local minima.

We could not find enough studies with numerical examples in the literature. Some of author hides data and/or results. Since no other comparable orthogonal fitting hyperboloid application could be found in literature. Against this background, the purpose of the study is to give an orthogonal fitting hyperboloid with a numerical example. In this article, we demonstrate that the geometric fitting approaches, provides a more robust alternative then algebraic fitting-although it is computationally more intensive. The most time consuming part of fitting hyperboloid is the calculation of the shortest (orthogonal) distances between each point and the hyperboloid. When we look at literature in this regard, we often see studies about ellipsoidal distances. We can develop a distance finding algorithm for hyperboloid by simulating it. And we did so, but this process is a little more difficult than the ellipsoid. In hyperboloids, one or two semi-axis are negatives, which changes the order of magnitude of the hyperboloid semi-axis. In the literature on shortest distances from an ellipsoid we see the various studies: Eberly, ${ }^{7}$ Eberly, ${ }^{8}$ Feltens, ${ }^{9}$ Ligas, ${ }^{10}$ Bektas. ${ }^{11}$ For the solution Eberly ${ }^{7}$ gives a method that is based on sixth degree polynominal. He has benefited from the largest root of 6th degree polynomial. Feltens ${ }^{9}$ gives a vector-based iteration process for finding the point on the ellipsoid. Ligas ${ }^{8}$ claims his method turns out to be more accurate, faster and applicable than Feltens method. Bektas ${ }^{12}$ is a little more advanced than the Ligas. ${ }^{10}$ A recent work on the subject gives us useful information on this topic (the orthogonal distance to Hyperboloid) for detailed information on this subject refer to Bektas. ${ }^{13}$

\section{Design of hyperboloid}

Hyperboloid a geometric surface consisting of one sheet, or of two sheets separated by a finit distance who sections parallel to the three coordinate planes are hyperbolas or ellipses. The hyperboloid of one sheet is possibly the most complicated of all the quadric surfaces. For one thing, its equation is very similar to that of a hyperboloid of two sheets, which is confusing. For another, its cross sections are quite complex. The standard equation of an hyperboloid centered at the origin of a cartesian coordinate system and aligned with the axis is given below.

Let a hyperboloid be given with the three semi axis a, b, c (i.e., the lengths of the real long axis, real short axis and the imaginary axis respectively) see Figure 2.

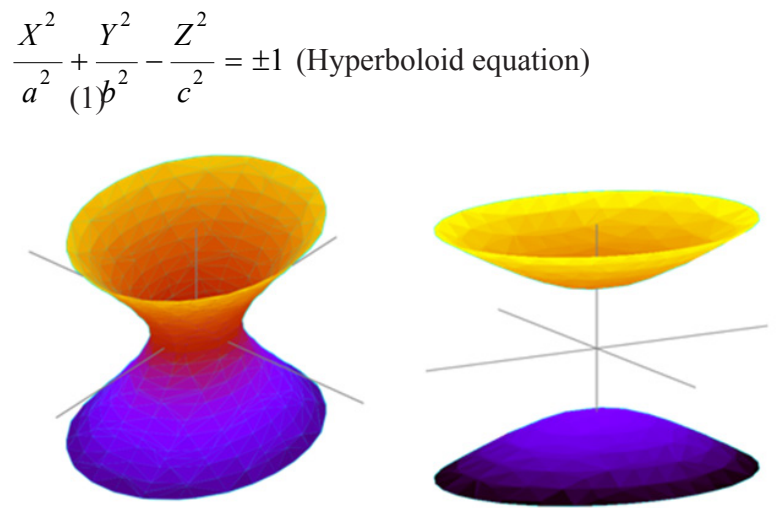

Figure 2 Hyperboloid: a) Hyperboloid of one sheet; b) Hyperboloid of two sheets.

+1 where on the right hand side of (1) corresponds to a hyperboloid of one sheet, on the right hand side of -1 to a hyperboloid of two sheets.

\section{Generalised equation of hyperboloid}

More generally, an arbitrarily oriented hyperboloid, centered at $\mathbf{v}$, is defined by the equation

$$
(x-v)^{T} A(x-v)=1
$$

where $A$ is a matrix and $x, v$ are vectors. The eigenvectors of A define the principal directions of the hyperboloid and the eigenvalues of $\mathrm{A}$ are the reciprocals of the squares of the semi-axis: $1 / a^{2}, 1 / b^{2}$ and $1 / c^{2}$. The one-sheet hyperboloid has two positive eigenvalues and one negative eigenvalue. The two-sheet hyperboloid has one positive eigenvalue and two negative eigenvalues Hilbert et al. ${ }^{14}$

\section{Fitting hyperboloid to noisy data}

Although hyperboloid equation is quite simple and smooth but computations are quite difficult on the hyperboloid. Generally a hyperboloid is defined 9 parameters. These parameters are; three coordinates of center $\left(X_{o}, Y_{o}, Z_{o}\right)$ three semi-axis $\left(\mathrm{a}\left(a_{x}, a_{y}, b\right)\right.$ ,and three rotational angles $\varepsilon, \psi, \omega$ which represent rotations around 
$\mathrm{x}-, \mathrm{y}-$ and $\mathrm{z}-$ axis respectively (Figure 2). These angles control the orientation of the hyperboloid. For this minimization problem to have a unique solution the necessary conditions is to be $n>=9$ and the data points lie in general position (e.g., not all data points should lie is an elliptic plane). Throughout this paper, we assume that these conditions are satisfied. For the solution of the fitting problem, the linear or linearized relationship, written between the given data points and unknown parameters (one equation per data points), consists of equations, including unknown parameters Bektas. ${ }^{13}$

\section{Algebraic direct hyperboloid fitting methods}

Algebraic direct fitting methods are a standard class of methods commonly used for fitting quadric surfaces. Algebraic fitting uses a generalized eigenvalue method.

The general equation of a hyperboloid is given as

$$
A x^{2}+B y^{2}+C z^{2}+2 D x y+2 E x z+2 F y z+2 G x+2 H y+2 I z-1
$$

Algebraic methods is a linear LS problem. It is solving directly easily. Given the data set $((\mathrm{x}, \mathrm{y}, \mathrm{z}) \mathrm{i}, \mathrm{i}=1,2, \ldots, \mathrm{n})$, the fitted hyperboloid by obtaining the solution in the LS sense of the linear algebraic equations.

$\mathrm{i}^{\text {th }}$ row of the nx9 matrix $\Omega$

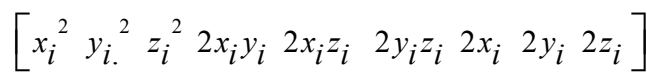

it is solved easily in the LS sense as below or it is solved easily as MATLAB expression:

$$
\begin{aligned}
& p=\left[\begin{array}{lllllll}
x^{2} y^{2}{ }^{2}{ }^{2}{ }^{2} & 2 x . y .2 x . z . & 2 y . z .2 x & 2 y & 2 z
\end{array}\right] \backslash \operatorname{ones}(n) \\
& \operatorname{ones}(\mathrm{n})=\left[\begin{array}{llll}
1 & 1 & \ldots & 1
\end{array}\right]^{\mathrm{T}}
\end{aligned}
$$

This fitting algorithm, we need to check whether a fitted shape is a hyperboloid. In theory, the conditions that ensure a quadratic surface to be a hyperboloid have been well investigated and explicitly stated in analytic geometry textbooks. Since a hyperboloid can be degenerated into other kinds of elliptic quadrics, such as an elliptic paraboloid. Therefore a proper constraint must be added Bektas. ${ }^{15}$

$$
\mathrm{m}=\left[\begin{array}{lll}
A & D & E \\
D & B & F \\
E & F & C
\end{array}\right] \mathrm{M}=\left[\begin{array}{cccc}
A & D & E & G \\
D & B & F & H \\
E & F & C & I \\
G & H & I & -1
\end{array}\right]
$$
of

$\rho_{3}=\operatorname{rank} \mathrm{m} ; \rho_{4}=\operatorname{rank} \mathrm{M} ; \Delta=\operatorname{det} \mathrm{M}$ And $\mathrm{k} 1, \mathrm{k} 2$ and $\mathrm{k} 3$ are the roots

$$
\left|\begin{array}{ccc}
A-x & D & E \\
D & B-x & F \\
E & F & C-x
\end{array}\right|=0
$$

The following conditions must be met For hyperboloid Beyer. ${ }^{16}$

$\rho_{3}=3, \rho_{4}=4, \operatorname{sign}(\Delta)=+$ and at least one of the signs of roots (k) must be different. In this paper, we assume that these conditions are satisfied. Algebraic methods all have indisputable advantage of solving linear LS problem. The methods for his are well known and fast. However, it is intuitively unclear what it is we are minimizing geometrically Eq (3) is often referred to as the "algebraic distance" to be minimized Ray et al. ${ }^{17}$

\section{Orthogonal (geometric) hyperboloid fitting methods}

Given the coordinates of $n$ points $x_{i}=\left[x_{i} y_{i} z_{i}\right], i=1,2, \ldots, n$, one needs to determine the optimal 9 parameters $p$ of an hyperboloid whose points $x$ satisfy the equation Eq.(3). This is a problem with 3n-9 degrees of freedom since once the parameters $p$ have been chosen, the shape and size of the hyperboloid ( 3 parameters), the position of its center ( 3 parameters) and the orientation of its axis ( 3 parameters) is completely defined and so are the coordinates $x_{i}{ }^{H}=\left[x_{i}{ }^{H} y_{i}^{H} z_{i}^{H}\right]$ of the projections of the corresponding points xi on the hyperboloid surface. The quantity (target function) to be minimized Bektas. ${ }^{12}$ To overcome the problems with the algebraic distances, it is natural to replace them by the orthogonal distances which are invariant to transformations in Euclidean space and which do not exhibit the high curvature bias. An hyperboloid of best fit in the LS sense to the given data points can be found by minimizing the sum of the squares of the shortest distances from the data to the hyperboloid . The geometric (shortest,orthogonal) distance is defined to be the distance between a data point and its closest point on the hyperboloid. For detailed information on this subject refer to Bektas. ${ }^{15}$ The following link can be used for the shortest distance and projection coordinates on hyperboloid URL$5 .{ }^{18}$ In this paper, we present techniques for hyperboloid fitting which are based on minimizing the sum of the squares of the geometric distances between the data and the hyperboloid. Determining best fit hyperboloid is a nonlinear least squares problem which in principle can be solved by using the Levenberg-Marquardt (LM) algorithm Levenberg, ${ }^{19}$ Marquardt. ${ }^{20}$ Generally, non-linear least squares is a complicated issue. It is very difficult to develop methods which can find the global minimizer with certainty in this situation. When a local minimizer has been discovered, we do not know whether it is a global minimizer or one of the local minimizer Zisserman. ${ }^{21}$ There are a variety of nonlinear optimization techniques, such as Newton, Gauss-Newton, Gradient Descent, Levenberg-Marquardt approximation etc. However, these fitting techniques, such as involve a highly nonlinear optimization procedure, which often stops at a local minimum and cannot guarantee an optimal solution Li et al..$^{22}$ Away from the minimum, in regions of negative curvature, the GaussNewton approximation is not very good. In such regions, a simple steepest-descent step is probably the best plan.

\section{Levenberg-marquardt algorithm}

The Levenberg-Marquardt algorithm is a blend of the Gradient descent and the Gauss-Newton iteration approaches designed for minimizing a target function of the form

$$
\phi(p)=\sum_{\mathrm{i}=1}^{\mathrm{n}}\left(y_{i}-f_{i}(p)\right)^{2}=(y-f(p))^{T}(y-f(p))=\min .
$$

or its "weighted" generalization

$$
\phi(p)=(y-f(p))^{T} W(y-f(p))=\min .
$$

As in both the Gradient descent and Gauss-Newton approach, it utilizes the Jacobian matrix $\mathrm{J}=\partial \mathrm{f} / \partial \mathrm{p}$ in order to minimize $\phi(p)=$ $\mathrm{e}^{\mathrm{T}} \mathrm{e}=\min$. where $\mathrm{e}=\mathrm{y}-\phi(p)$.

Now if we set in our case $f_{i}=\psi\left(x_{i}^{H}, p\right)$ we can solve the algebraic problem directly but not the geometric one, because the latter is based 
on the constraints $\mathrm{f}_{\mathrm{i}}=\psi\left(\mathrm{x}_{\mathrm{i}}^{\mathrm{H}}, \mathrm{p}\right)$ and also the missing "projection" constrains securing that $\mathrm{x}_{\mathrm{i}}^{\mathrm{H}}$ is the projection of $\mathrm{xi}$ on the hyperboloid . The Levenberg-Marquardt involves the modified Hessian

$$
H(p, \lambda)=J^{T} J+\lambda I(\mathrm{I} \text { : identity matrix })
$$

in order to compute iteratively corrections $\delta \mathrm{p}$

which update the values of $\mathrm{p}$ until $\phi(p)=$ min is achieved. The linearization

$$
f\left(p_{O}+d p\right)=f\left(p_{O}\right)+\left(p_{O}\right) d p=f\left(p_{O}\right)+J\left(p_{O}\right) d p
$$

plays a crucial role in the algorithm formulation.

The LM algorithm does not require explicit line searches. More iterations than Gauss-Newton, but, no line search required, and more frequently converge suppose that we have a unknowns parameter set

$$
p=[A B C D E F G H I]^{T}
$$

Unknown the conic parameters. The general conic equation an hyperboloid is given as Eq.(3)

We will go to solution establishing relationships between the conical coefficient variations with the orthogonal distances. The initial parameters were derived from the algebraically fitting hyperboloid

$\mathrm{x}_{\mathrm{i}}^{\mathrm{H}} \mathrm{y}_{\mathrm{i}}^{\mathrm{H}} \mathrm{z}_{\mathrm{i}}^{\mathrm{H}}$ : Projection coordinates (onto hyperboloid) of given Pi data points

ith row of the nx10 matrix $\mathrm{J}$ (Jakobian matrix)

$$
\left[\begin{array}{llllllllll}
x_{i}^{2} & y_{i}^{2} & z_{i}^{2} & 2 x_{i} y_{i} & 2 x_{i} z_{i} & 2 y_{i} z_{i} & 2 x_{i} & 2 y_{i} & 2 z_{i} & -1
\end{array}\right]
$$

ith row of the right side vector $h_{n \times 1}$

$$
h_{i}=\operatorname{sign}\left(\mathrm{z}_{\mathrm{i}}-\mathrm{z}_{\mathrm{i}}^{H}\right) \operatorname{sign}\left(\mathrm{z}_{\mathrm{i}}^{\mathrm{H}}\right) \sqrt{\left(x_{\mathrm{i}}^{\mathrm{H}}-\mathrm{x}_{\mathrm{i}}\right)^{2}+\left(y_{\mathrm{i}}^{\mathrm{H}}-\mathrm{y}_{\mathrm{i}}\right)^{2}+\left(\mathrm{z}_{\mathrm{i}}^{\mathrm{H}}-\mathrm{z}_{\mathrm{i}}\right)^{2}}
$$

We obtained below linearized equation

$$
\begin{aligned}
& \mathrm{J}_{\mathrm{nx} 10} \cdot d p_{n x 1}=h_{n x 1} \\
& d p=\left[\begin{array}{lllllllll}
d A & d B & d C & d D & d E & d F & d G & d H & d I
\end{array}\right]^{T}
\end{aligned}
$$

The fitted orthogonal hyperboloid by obtaining the solution in the LS sense with L-M algorithm Bektas. ${ }^{10}$

\section{The Levenberg-Marquardt algorithm in pseudo-code}

1-Solve algebraic methods and find initial values for $\mathrm{p}$

set $\lambda=1$ (say)

2- Compute J- Jacobian matrix and hi orthogonal distances from all given data points

$$
\begin{gathered}
\operatorname{minh}=h^{T} h \\
\text { 3- Solve }\left(J^{T} J+\lambda I\right) d p=J^{T} h \\
p=p+d p \text {, new conic parameter }
\end{gathered}
$$

Find again hi orthogonal distances from all given data points

newh $=h^{T} h$

4- if newh $<\operatorname{minh} \%$ yes there is improvement, reduce $\lambda$

$\operatorname{minh}=$ newh $\lambda \lambda=\lambda / 2 \operatorname{minh}=$ newh $; \lambda=\lambda / 2$ goto 3

else $\%$ no improvement, increase $\lambda$

$\lambda=2 * \lambda$

goto 3

end

\section{Obtaining hyperboloid parameters from conic equation}

After conical equation Eq.(3) was determined. This section we determines the center, semi-axis and rotation angles of the hyperboloid.

$$
\left[\begin{array}{ccc}
A & D & E \\
D & B & F \\
E & F & C
\end{array}\right]\left[\begin{array}{c}
X_{O} \\
Y_{O} \\
Z_{O}
\end{array}\right]+\left[\begin{array}{c}
G \\
H \\
I
\end{array}\right]=\left[\begin{array}{l}
0 \\
0 \\
0
\end{array}\right]
$$

The solution of above equation system which is established conic coefficients gives us the coordinates of hyperboloid's center $\left(X_{o}, Y_{o}, Z_{o}\right)$.

For finding of semi-axis $(a, b, c)$ and rotation angles of the hyperboloid $\varepsilon, \psi, \omega$ :

Firstly eigenvalues and eigenvectors of above coefficient matrix (S3x3) in Eq (17) can be easily calculated with the following MATLAB command

[ evecs, evals] $=\operatorname{eig}(\mathrm{S})$

$$
\text { evals: Eigenvalues of }(S)=\left[\begin{array}{lll}
l_{1} & l_{2} & l_{3}
\end{array}\right]^{\mathrm{T}}
$$

Let 11,12 and 13 get the eigenvalues of the matrix $\mathrm{S}$, in descending order Semi-axis of hyperboloid $(a, b, c)$ obtained the eigenvalues of $\mathrm{S}$ as below

$$
\begin{gathered}
\mathrm{a}=\operatorname{sign}\left(\lambda_{1}\right) / \sqrt{\operatorname{abs}\left(\lambda_{1}\right)} \quad \mathrm{b}=\operatorname{sign}\left(\lambda_{2}\right) / \sqrt{a b s\left(\lambda_{2}\right)} \\
\mathrm{c}=\operatorname{sign}\left(\lambda_{3}\right) / \sqrt{a b s\left(\lambda_{3}\right)}
\end{gathered}
$$

It should not be forgotten that the one-sheet hyperboloid has two positive eigenvalues and one negative eigenvalue. The twosheet hyperboloid has one positive eigenvalue and two negative eigenvalues. So it is necessary to pay attention to this in the rooting process. Rooting can be done as above.

evecs: Eigenvectors of (S) give us R - rotation angles of hyperboloid.

The following link can be used for hyperboloid parameter from the conic equation URL-6. ${ }^{23}$

\section{Numerical example and discussion}

For numerical applications 20 triplets $(\mathrm{x}, \mathrm{y}, \mathrm{z})$ cartesian coordinates were produced. Here data point's coordinates and results as shown Tables $1 \& 2$. 
Table I The result of direct fitting hyperboloid

\begin{tabular}{|c|c|c|c|c|c|c|c|}
\hline \multicolumn{3}{|c|}{ Center of coordinates } & \multicolumn{3}{|c|}{ Semi-axis } & \multirow[t]{2}{*}{ Rotational matrix } & \multirow[t]{2}{*}{ RSS* } \\
\hline xo & yo & zo & $\mathbf{a}$ & b & c & & \\
\hline \multirow[t]{2}{*}{0.65706} & 0.44483 & -8.9262 & 3.2408 & 8.0121 & $-|0.568|$ & $0.00780 .0074-1$ & 1.4721 \\
\hline & & & & & & $0.0115|0.0075|-0$. & 077 \\
\hline
\end{tabular}

Parameter of algebraic direct fitting: 0.057450 .00940 I3 $-0.0053975-0.00036$ I $-0.00048776-0.000$ I 68 -0.04I94I $-0.0054448-0.047784$

Table 2 The result of orthogonal fitting hyperboloid

\begin{tabular}{|c|c|c|c|c|c|c|c|}
\hline \multicolumn{3}{|c|}{ Center of coordinates } & \multicolumn{3}{|c|}{ Semi-axis } & \multirow[t]{2}{*}{ Rotational matrix } & \multirow[t]{2}{*}{ RSS* } \\
\hline xo & yo & zo & $\mathbf{a}$ & b & c & & \\
\hline \multirow[t]{3}{*}{0.6288} & 0.23378 & -9.2781 & 3.1863 & 7.6387 & $-|0.578|$ & $0.01020 .0074-1$ & 1.1027 \\
\hline & & & & & & $0.0242 \mid 0.0076$ & \\
\hline & & & & & & $1-0.02430 .0100$ & \\
\hline
\end{tabular}

*RSS:The residual sum of squares of the orthogonal distances

Find the best fitting hyperboloid for the given noisy data is based on both of algebraic and orthogonal methods.

$$
\begin{aligned}
& \text { x: }-4-4-4-4-4-4-4-2-2-2-21114444444
\end{aligned}
$$

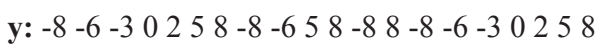

Table 3 Orthogonal fitting result L-M results z: $66433461-4-9-2-2-73-1-4-7-6-31$

We show both the algebraic and orthogonal fitting results are as shown in Tables $1 \& 3$ (Figure 3).

\begin{tabular}{|c|c|c|c|c|c|c|c|c|c|c|c|c|}
\hline İter. & $\lambda$ & $\mathbf{A}$ & B & C & D & $\mathbf{E}$ & $\mathbf{F}$ & G & $\mathbf{H}$ & I & RSStemp & RSS \\
\hline I & I & 0.05348 & 0.01504 & -0.00942 & -0.00123 & -0.00424 & -0.00088 & -0.05366 & -0.01045 & -0.06366 & 9.2212 & 1.4721 \\
\hline I & 2 & 0.05344 & 0.01504 & -0.00937 & -0.00122 & -0.00423 & -0.00088 & -0.05349 & -0.01044 & -0.06349 & 9.1964 & $1.472 \mathrm{I}$ \\
\hline I & 4 & 0.0533 & 0.01501 & -0.00918 & -0.0012 & -0.00418 & -0.00086 & -0.05283 & -0.01039 & -0.06286 & 9.1025 & 1.4721 \\
\hline I & 8 & 0.05292 & 0.01493 & -0.00859 & -0.00115 & -0.004 & -0.0008 & -0.05078 & -0.01021 & -0.06086 & 8.793 & $1.472 \mathrm{I}$ \\
\hline I & 16 & 0.05269 & 0.01469 & -0.00742 & -0.00101 & -0.00349 & -0.00068 & -0.04677 & -0.00972 & -0.05669 & 8.054 & $1.472 \mathrm{I}$ \\
\hline I & 32 & 0.05407 & 0.01421 & -0.00626 & -0.00081 & -0.0025 & -0.00058 & -0.04332 & -0.00867 & -0.05216 & 6.8853 & 1.4721 \\
\hline I & 64 & 0.05628 & 0.0135 & -0.00552 & -0.00062 & -0.00151 & -0.00054 & -0.04214 & -0.0072 & -0.04928 & 5.5602 & $1.472 \mathrm{I}$ \\
\hline I & 128 & 0.05739 & 0.01244 & -0.00504 & -0.00052 & -0.00105 & -0.00056 & -0.04197 & -0.00611 & -0.04816 & 3.829 & 1.4721 \\
\hline I & 256 & 0.05759 & 0.01104 & -0.00499 & -0.00042 & -0.00083 & -0.00054 & -0.04195 & -0.00565 & -0.04787 & $|.803|$ & 1.4721 \\
\hline I & 512 & 0.05752 & 0.01 & -0.0052 & -0.00037 & -0.00063 & -0.00037 & -0.04195 & -0.0055 & $-0.0478 \mid$ & I.1028 & 1.4721 \\
\hline 2 & 256 & 0.05772 & 0.01134 & -0.00475 & -0.00047 & -0.00098 & $-0.0005 I$ & -0.04199 & -0.00572 & -0.04787 & 2.329 & 1.1028 \\
\hline 2 & 512 & 0.05761 & 0.01049 & -0.00502 & -0.00039 & -0.00077 & -0.00046 & -0.04196 & -0.00556 & -0.04782 & 1.3134 & 1.1028 \\
\hline 2 & 1024 & 0.05755 & 0.01014 & $-0.005 \mid 5$ & -0.00037 & -0.00067 & -0.0004 & -0.04195 & $-0.0055 I$ & -0.04781 & 1.1239 & 1.1028 \\
\hline 2 & 2048 & 0.05753 & 0.01004 & -0.00519 & -0.00037 & -0.00064 & -0.00037 & -0.04195 & -0.0055 & $-0.0478 \mid$ & 1.1049 & I.1028 \\
\hline 2 & 4096 & 0.05753 & 0.01001 & -0.0052 & -0.00037 & -0.00063 & -0.00037 & -0.04195 & -0.0055 & -0.04781 & 1.1031 & I.1028 \\
\hline 2 & 8192 & 0.05753 & 0.01001 & -0.0052 & -0.00037 & -0.00063 & -0.00037 & -0.04195 & -0.0055 & $-0.0478 \mid$ & I.1028 & I.1028 \\
\hline
\end{tabular}

Parameter of orthogonal fitting: $0.0575240 .010004-0.0052-0.00036-0.00063-0.00036-0.0419-0.0055-0.047807-1.0$ 


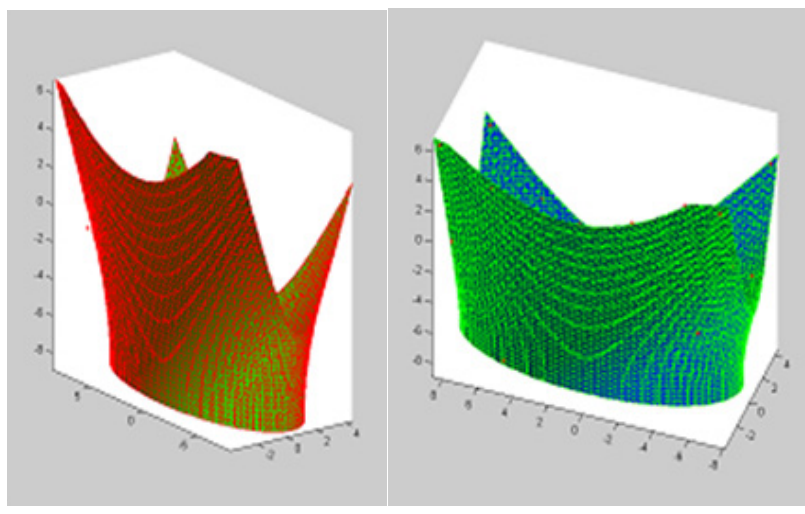

Figure 3 Fitting result: a) Algebraic direct fitting; b) Orthogonal fitting.

\section{Discussion and suggestions}

Orthogonal least-squares have a much sounder basis, but is usually difficult to implement. Why are algebraic distances usually not satisfactory? The big advantage of use of algebraic distances is the gain in computational efficiency, because closed-form solutions can usually be obtained. In general, however, the results are not satisfactory. The function to minimize is usually not invariant under Euclidean transformations. This is a feature we dislike, because we usually do not know in advance where the best coordinate system to represent the data is. A point may contribute differently to the parameter estimation depending on its position on the conic. If a priori all points are corrupted by the same amount of noise, it is desirable for them to contribute the same way. ${ }^{24}$ More importantly, algebraic methods have an inherent curvature bias-data corrupted by the same amount of noise will misfit unequally at different curvatures. ${ }^{17}$ It is also seen in our numerical application that orthogonal fitting improves the results. The RSS indicator, which is a quality indicator, decreased to 1,1072 from 1,4721 . Our experience tells us that if the coordinates of given points consists of a large number this will cause bad condition. Therefore, before fitting, you must shift the given coordinates to the center of gravity, after fitting operation the coordinates of hyperboloid's center must be shifted back to the previous position. In this study, there is no mention of the accuracy (variances) of the parameters obtained from the fitting and, consequently, of the accuracies of the estimated geometric elements of the hyperboloid. Because the geometric elements of the hyperboloid are obtained indirectly. We have achieved first conical parameters after that the geometric parametric of the hyperboloid. Hence the accuracy calculations require long study. Therefore, we consider the accuracy calculations can be subject a separate subject study. ${ }^{25-29}$

\section{Conclusion}

In this paper we study on the orthogonal fitting hyperboloid. From the results, it is apparent that the orthogonal fitting hyperboloid always exhibits less RSS error than the algebraic direct fitting hyperboloid. The problem of fitting hyperboloid is encountered frequently in the image processing, In the modeling of some industrial parts, computer games, etc. The paper has presented a new method of orthogonal fitting hyperboloid. The new method relies on solving an over determined system of nonlinear equations with the use of L-M method. It has been compared to the other existing methods. In conclusion, the presented method may be considered as fast, accurate and reliable and may be successfully used in other areas. The presented orthogonal fitting algorithm can be applied easily for ellipsoid, and sphere also other surface such as paraboloid. In our future research, we plan to focus different estimation technique such as the 11-norm method. The disadvantages of the LS (the 12-norm) method are that is affected by noisy data (outliers, gross errors) and it distributes to the sensitivity measurements. In this case, hyperboloid fitting is a very nice application. With LS techniques, even one or two outliers in a large set can wreak havoc! Outlying data give an effect so strong in the minimization that the parameters thus estimated by those outlying data are distorted. Numerous studies have been conducted, which clearly show that least-squares estimators are vulnerable to the violation of these assumptions. Sometimes, even when the data contains only one bad measurement, LS method estimates may be completely perturbed. The solution of the 11-norm method is not always unique, and there may be several solutions. Also, the solution of the 11-norm method is not generally obtained directly, but iteratively calculations are made. Therefore, the solution is not easily calculated like in the 12-norm method. Notwithstanding, when the computational tools, computer capacity and speed are considered, the difficulty of calculations are eliminated. The advantages of the 11-norm method are non-sensitivity against measurements, including gross errors, and the solution is not or is little affected by these measurements.

\section{Acknowledgments}

None.

\section{Conflicts of interest}

The author declares there are no conflicts of interest.

\section{References}

1. Hyperboloid Structures in GSA.

2. Applications of Hyperbolas. 2012.

3. http://conicsectionjpg.blogspot.com.tr/2012/10/applications-ofhyperbola.html

4. Min D, TS Newman. Hyperbolic and Parabolic Quadric Surface Fitting Algorithms-Comparison Between the Least Squares Approach and the Parameter Optimization Approach. 1998.

5. Andrews J, Séquin CH. Type-Constrained Direct Fitting of Quadric Surfaces. Computer-Aided Design \& Applications. 2013.

6. EL Hall, JBK Tio, CA McPherson, et al. Measuring Curved Surfaces for Robot Vision. IEEE Computer. 1982;15(12):42-54.

7. X Cao, N Shrikhande, G Hu. Approximate orthogonal distance regression method for fitting quadric surfaces to range data. Pattern Recognition Letters. 1994;15(8):781-796.

8. Eberly D. Least Squares Fitting of Data. Geometric Tools, LLC. 2008.

9. Eberly D. Distance from a Point to an Ellipse, an Ellipsoid, or a Hyperellipsoid. Geometric Tools, LLC, USA. 2013.

10. Feltens J. Vector method to compute the Cartesian $(X, Y, Z)$ to geodetic $(\mathrm{j}, \lambda, h)$ transformation on a triaxial ellipsoid. Journal of Geod. 2009;83(2):129-137.

11. Ligas M. Cartesian to geodetic coordinates conversion on a triaxial ellipsoid. Journal of Geod. 2012;86(4):249-256.

12. Bektas S. Orthogonal distance from an ellipsoid. 2014.

13. Bektas S. Orthogonal (shortest) distance from an hyperboloid. International Journal of Research in Engineering and Applied Sciences. 2017;7(5):37-45. 
14. Hilbert D, Cohn Vossen S. The Second-Order Surfaces. 3 in Geometry and the Imagination. New York: Chelsea, USA. 1999;12-19.

15. Bektas S. Least squares fitting of ellipsoid using orthogonal distances. Boletim de Ciencias Geodesicas. 2015;21(2):1982-2170.

16. Beyer WH. CRC Standard Mathematical Tables 28th edn. Boca Raton, FL: CRC Press, USA. 1987;210-211.

17. Ray A, Srivastava DC. Non-linear least squares ellipse fitting using the genetic algorithm with applications to strain analysis. Journal of Structural Geology. 2008;30(2):1593-1602.

18. Sebahattin Bektas. Shortest_distance (X axis). 2017.

19. Levenberg K. A method for the solution of certain problems in least squares. Quart Appl Math. 1944;2(2):164-168.

20. Marquardt D. An algorithm for least-squares estimation of nonlinear parameters. SIAM J Appl Math. 1963;11(2):431-441.

21. Zisserman A. C25 Optimization, 8 Lectures. Hilary Term, 2 Tutorial Sheets, Lectures 3-6 (BK). 2013.
22. Li Q, Griffiths JG. Least Squares Ellipsoid Specific Fitting. Proceedings of the Geometric Modelling and Processing. 2004.

23. Sebahattin Bektas. Conversion from conic parameters to geometric parameters of ellipsoids. Math works. 2017.

24. Zhang Z. Parameter estimation techniques: a tutorial with application to conic fitting. Image and Vision Computing. 1997;15(1):59-76.

25. Sebahattin Bektas, Faik Ahmet Sesli, Erdi Pakel. Orthogonal Shortest Distance To the Hyperboloid. International Journal of Research in Engineering and Applied Sciences. 2017;7(5):37-45.

26. Chernov N, Ma H. Least squares fitting of quadratic curves and surfaces, Computer Vision, USA. 2011;285-302.

27. Mollin RA. Quadrics. Boca Raton, FL: CRC Press, USA. 1995.

28. The Hyperboloid and its Applications to Engineering.

29. For hyperbola and hyperboloid applications. 